\title{
TSYGAN FORMALITY AND DUFLO FORMULA
}

\author{
BORIS SHOIKHET
}

\begin{abstract}
We prove the 0-(co)homology part of the conjecture on the cupproducts on tangent cohomology in the Tsygan formality [Sh2]. We discuss its applications to the Duflo formula.
\end{abstract}

\section{A short introduction}

The Tsygan formality conjecture for chains [Ts] was proven in the author's work [Sh2] by an explicit construction of suitable Kontsevich-type integrals. This paper is a further development of ideas of [Sh2]. We will freely use the notations and results of [Sh2]. In [Sh2] we formulated a conjecture on "the cup-products on tangent cohomology", which is a version of the analogous Kontsevich theorem from Section 8 of $[\mathrm{K}]$. Here we prove this conjecture for 0-(co)homology.

\section{The classical Duflo formula and the generalized Duflo formula}

1.1. Let $\mathfrak{g}$ be a finite-dimensional Lie algebra, $S^{\bullet}(\mathfrak{g})$ and $U(\mathfrak{g})$ be its symmetric and universal enveloping algebra. They are not isomorphic as algebras $S^{\bullet}(\mathfrak{g})$ is a commutative algebra and $U(\mathfrak{g})$ is a non-commutative algebra. We can consider both spaces $S^{\bullet}(\mathfrak{g})$ and $U(\mathfrak{g})$ as $\mathfrak{g}$-modules with the adjoint action for $S^{\bullet}(\mathfrak{g})$ and the action $g \cdot \omega=g \otimes \omega-\omega \otimes g$ for $U(\mathfrak{g})$ (here $g \in \mathfrak{g}$ and $\omega \in U(\mathfrak{g})$ ). It is clear that these $g$-modules are isomorphic, the isomorphism is the classical Poincaré-Birkhoff-Witt map:

$$
\varphi_{P B W}\left(g_{1} \cdots g_{k}\right)=\frac{1}{k !} \sum_{\sigma \in \Sigma_{k}} g_{\sigma(1)} \otimes \cdots \otimes g_{\sigma(k)}
$$

$\left(g_{1}, \ldots, g_{k} \in \mathfrak{g}\right)$.

The Duflo theorem $[\mathrm{D}]$ states that the invariants $\left[S^{\bullet}(\mathfrak{g})\right]^{\mathfrak{g}}$ and $[U(\mathfrak{g})]^{\mathfrak{g}}$ are isomorphic as algebras. The Duflo formula is a canonical formula for this isomorphism. We recall it here.

For any $k \geq 1$, there exists a canonical element in $\left[S^{k}\left(\mathfrak{g}^{*}\right)\right]^{\mathfrak{g}}$. It is the symmetrization of the map

$$
\left.g \mapsto \operatorname{Tr}\right|_{\mathfrak{g}} \operatorname{ad}^{k} g \quad(g \in \mathfrak{g}) .
$$

We denote this element in $\left[S^{k}\left(\mathfrak{g}^{*}\right)\right]^{\mathfrak{g}}$ by $\operatorname{Tr}_{k}$. We can consider an element from $S^{k}\left(\mathfrak{g}^{*}\right)$ as a differential operator of the $k$-th order with constant coefficients, acting on $S^{\bullet}(\mathfrak{g})$. (Thus, an element from $\mathfrak{g}^{*}$ is a derivation of $S^{\bullet}(\mathfrak{g})$ ). (It was

Received December 14, 2000. 
a conjecture of M. Duflo that the operators corresponding to $S^{k}\left(\mathfrak{g}^{*}\right)^{\mathfrak{g}}$ are zero for odd $k$ and any finite-dimensional Lie algebra $\mathfrak{g}$; this conjecture was proven recently in $[\mathrm{AB}])$.

Define the map $\varphi_{\text {strange }}: S^{\bullet}(\mathfrak{g}) \rightarrow S^{\bullet}(\mathfrak{g})$ by the formula:

$$
\varphi_{\text {strange }}=\exp \left(\sum_{k \geq 1} \alpha_{2 k} \cdot \operatorname{Tr}_{2 k}\right)
$$

where

$$
\sum_{k \geq 1} \alpha_{2 k} \cdot x^{2 k}=\frac{1}{2} \log \frac{e^{\frac{x}{2}}-e^{-\frac{x}{2}}}{x} .
$$

The map $\varphi_{\text {strange }}$ is well-defined on $S^{\bullet}(\mathfrak{g})$ (in the sense that we have no problems with divergences), because $\operatorname{Tr}_{2 k}(\omega) \equiv 0$ for a fixed $\omega \in S^{\bullet}(\mathfrak{g})$ and for a sufficiently large $k$. The map $\varphi_{\text {strange }}$ is a map of $\mathfrak{g}$-modules, because the operators $\operatorname{Tr}_{2 k}$ are invariant.

Theorem (M. Duflo, [D]). The restriction of the map $\varphi_{D}=\varphi_{P B W} \circ \varphi_{\text {strange }}$ to the invariants $\left[S^{\bullet}(\mathfrak{g})\right]^{\mathfrak{g}}$ defines a map of algebras $\varphi_{D}:\left[S^{\bullet}(\mathfrak{g})\right]^{\mathfrak{g}} \rightarrow[U(\mathfrak{g})]^{\mathfrak{g}}$.

1.1.1. M. Kontsevich deduced from his theorem on cup-products on the tangent cohomology $[\mathrm{K}]$ the following generalization of the Duflo theorem.

Theorem. There exists a canonical map $\tilde{\varphi_{D}}: H^{\bullet}\left(\mathfrak{g} ; S^{\bullet}(\mathfrak{g})\right) \rightarrow H^{\bullet}(\mathfrak{g} ; U(\mathfrak{g}))$ which is a map of associative algebras. Its restriction to $H^{0}\left(\mathfrak{g} ; S^{\bullet}(\mathfrak{g})\right)$ coincides with the Duflo map $\varphi_{D}$. This result holds also for any $\mathbb{Z}$-graded finitedimensional Lie algebra $\mathfrak{g}$.

Recall, that $M^{\mathfrak{g}}=H^{0}(\mathfrak{g} ; M)$ for any $\mathfrak{g}$-module $M$. This map $\tilde{\varphi_{D}}$ is given as the tangent map to the Kontsevich $L_{\infty}$ formality morphism at the solution to the Maurer-Cartan equation corresponding to the Kostant-Kirillov Poisson structure on $\mathfrak{g}^{*}$. In the case of $H^{0}\left(\mathfrak{g} ; S^{\bullet}(\mathfrak{g})\right)$ this tangent map can be computed (not so easy, by comparing with the Duflo formula for $g l_{N}$ in the Kontsevich original approach). In this case all the graphs are unions of so called wheels. For higher cohomology $H^{k}\left(\mathfrak{g} ; S^{\bullet}(\mathfrak{g})\right), k \geq 1$, many other graphs besides the wheels appear, and it seems that any computation of the Feynmann(Kontsevich) weights of these other graphs is impossible. Nevertheless, we can prove the following result.

\subsubsection{1. .}

Theorem. Denote by $\varphi_{D}^{\bullet}: H^{\bullet}\left(\mathfrak{g} ; S^{\bullet}(\mathfrak{g})\right) \rightarrow H^{\bullet}(\mathfrak{g} ; U(\mathfrak{g}))$ the map induced by the map of $\mathfrak{g}$-modules $\varphi_{D}: S^{\bullet}(\mathfrak{g}) \rightarrow U(\mathfrak{g})$. Then the map $\varphi_{D}^{\bullet}$ is a map (an isomorphism) of associative (graded commutative) algebras.

Proof. We just sketch the proof here. The complete proof will appear somewhere else. This proof is based on an unpublished joint paper with Maxim Kontsevich.

Consider the space $V=\mathfrak{g}[1]$. It is a $\mathbb{Z}$-graded vector space. Consider the $L_{\infty}$ formality morphism on it. The polyvector fields $T_{\text {poly }}(V)$ is isomorphic 
to $T_{\text {poly }}\left(\mathfrak{g}^{*}\right) \simeq \wedge^{\bullet}\left(\mathfrak{g}^{*}\right) \otimes S^{\bullet}(\mathfrak{g})$. (This phenomenon can be considered roughly as a kind of the Koszul duality). There is an odd vector field $Q$ on $\mathfrak{g}[1]$ such that $Q^{2}=0$ (which generates the cochain differential). In coordinates, $Q=$ $\sum_{i, j, k=1}^{\operatorname{dim} \mathfrak{g}} c_{i j}^{k} \xi_{i} \xi_{j} \frac{\partial}{\partial \xi_{k}}$ where $c_{i j}^{k}$ are the structure constants of the Lie algebra $\mathfrak{g}$ in some basis $x_{i}$ and $\xi_{i}$ are the odd coordinates on $\mathfrak{g}[1]$ corresponding to $x_{i}$.

We want know to localize the formality morphism on $V$ at the solution to the Maurer-Cartan equation $Q \in\left[T_{\text {poly }}(V)\right]^{1}$. We claim that the only graphs which appear are unions of the wheels.

It follows from $[\mathrm{K}]$, Lemma7.3.3.1(1). Note that here the wheels are not the same wheels as for $\mathfrak{g}^{*}$ : here we have one outgoing edge and two incoming edges for each vertex whence for $\mathfrak{g}^{*}$ we have two outgoing edges and one incoming. It reflects the fact that $Q$ is a (quadratic) vector field whence the Kostant-Kirillov Poisson structure $\alpha=\sum_{i, j, k=1}^{\operatorname{dim} \mathfrak{g}} c_{i j}^{k} x_{k} \frac{\partial}{\partial x_{i}} \wedge \frac{\partial}{\partial} \partial x_{j}$ is a (linear) bivector field.

It is not straightforward to compute the Kontsevich weights of these wheels corresponding to $\mathfrak{g}[1]$ but it turns out it is possible. The answer is exactly the formula in the Theorem.

Later in this paper we consider only the case of 0-th cohomology, to simplify the exposition. While the Kontsevich claim on the cup-products was proved recently for higher cohomology in $[\mathrm{MT}]$, in the case of Tsygan formality we prove the corresponding theorem on cup-products for 0-cohomology only. It seems, however, that the technique developed in $[\mathrm{MT}]$ can be used in this situation.

1.2. In $[\mathrm{Sh} 2]$ we proposed the following conjecture:

Conjecture. Denote by $\varphi_{D \bullet}: H_{\bullet}\left(\mathfrak{g} ; S^{\bullet}(\mathfrak{g})\right) \rightarrow H_{\bullet}(\mathfrak{g} ; U(\mathfrak{g}))$ the map defined by the map of $\mathfrak{g}$-modules $\varphi_{D}: S^{\bullet}(\mathfrak{g}) \rightarrow U(g)$. Then the map $\varphi_{D \bullet}: H_{\bullet}\left(\mathfrak{g} ; S^{\bullet}(\mathfrak{g})\right) \rightarrow$ $H_{\bullet}(\mathfrak{g} ; U(\mathfrak{g}))$ is a map of modules from the $H^{\bullet}\left(\mathfrak{g} ; S^{\bullet}(\mathfrak{g})\right)^{\text {opp }}$-module $H_{\bullet}(\mathfrak{g} ; S(\mathfrak{g}))$ to the $\left(H^{\bullet}(\mathfrak{g} ; U(\mathfrak{g}))^{\text {opp }}\right.$-module $H_{\bullet}(\mathfrak{g} ; U(\mathfrak{g}))$. It means that for any $\alpha \in H^{\bullet}(\mathfrak{g} ; S(\mathfrak{g}))$ and any $\beta \in H^{\bullet}(\mathfrak{g} ; S(\mathfrak{g}))$ one has:

$$
\varphi_{D \bullet}(\alpha \triangleleft \beta)=\varphi_{D}^{\bullet}(\alpha) \star \varphi_{D \bullet}(\beta) .
$$

Here we denote by the canonical action of $\alpha \in H^{\bullet}\left(\mathfrak{g} ; S^{\bullet}(\mathfrak{g})\right)$ on $\beta \in$ $H_{\bullet}\left(\mathfrak{g} ; S^{\bullet}(\mathfrak{g})\right)$ and by $\star$ the action of $H^{\bullet}(\mathfrak{g}, U(\mathfrak{g}))$ on $H_{\bullet}(\mathfrak{g} ; U(g))$. As in general, cohomology forms an algebra, and homology forms a module over it.

Recall, that $H_{0}(\mathfrak{g} ; M)=M / \mathfrak{g} M=M_{\mathfrak{g}}$ is the space of coinvariants. For 0cohomology this conjecture states that $\left(S^{\bullet}(\mathfrak{g})\right)^{\mathfrak{g}}$-module $\left(S^{\bullet}(\mathfrak{g})\right)_{\mathfrak{g}}$ and $(U(\mathfrak{g}))^{\mathfrak{g}_{-}}$ module $(U(\mathfrak{g}))_{\mathfrak{g}}$ are isomorphic by means of the Duflo map $\varphi_{D}$.

1.2.1. We prove here the following statement:

Theorem. For any finite-dimensional Lie algebra $\mathfrak{g}$ (or any finite-dimensional $\mathbb{Z}$-graded Lie algebra $\mathfrak{g})$ one has:

$$
\varphi_{D}(\alpha \cdot \beta+c(\alpha, \beta))=\varphi_{D}(\alpha) \star \varphi_{D}(\beta)
$$

where $\alpha \in\left[S^{\bullet}(\mathfrak{g})\right]^{\mathfrak{g}}, \beta \in S^{\bullet}(\mathfrak{g})$, * is the product in $U(\mathfrak{g})$, and $c(\alpha, \beta) \in\left\{\mathfrak{g}, S^{\bullet}(\mathfrak{g})\right\}$. 
As well, we obtain an explicit formula for $c(\alpha, \beta)$. It is clear that this theorem implies Conjecture above for 0-(co)homology. For a semisimple Lie algebra $\mathfrak{g}$, this theorem is equivalent to the Duflo formula because of the decompositions

$$
\begin{aligned}
S^{\bullet}(\mathfrak{g}) & =\left[S^{\bullet}(\mathfrak{g})\right]^{\mathfrak{g}} \oplus\{S(\mathfrak{g}), S(\mathfrak{g})\}, \\
U(\mathfrak{g}) & =[U(\mathfrak{g})]^{\mathfrak{g}} \oplus[U(\mathfrak{g}), U(\mathfrak{g})] .
\end{aligned}
$$

which hold for any semisimple Lie algebra $\mathfrak{g}$.

For an arbitrary Lie algebra $\mathfrak{g}$, this theorem is not a corollary of the Duflo formula, and it is a new fact about the Duflo map.

\section{The theorem on cup-products in Tsygan formality}

Here we prove the conjecture on the cup-products in the Tsygan formality [Sh2] for 0-cohomology. We use the notations from [Sh2].

This conjecture is analogous to the Kontsevich theorem on a cup-products in $[\mathrm{K}]$, Section 8. It would be helpful for reader to know the Kontsevich's proof. It is proven for 0 -th tangent cohomology in $[\mathrm{K}]$, and in $[\mathrm{MT}]$ in the general case.

2.1. Recall that the Kontsevich $L_{\infty}$-morphism $\mathcal{U}: T_{\text {poly }}^{\bullet}\left(\mathbb{R}^{d}\right) \rightarrow \mathcal{D}_{\text {poly }}^{\bullet}\left(\mathbb{R}^{d}\right)$ (see $[\mathrm{K}])$ and the Lie derivatives $L_{\Psi}: C_{\bullet}(A, A) \rightarrow C_{\bullet}(A, A)$ allows to define a $T_{\text {poly }}^{\bullet}\left(\mathbb{R}^{d}\right)-L_{\infty}$-module structure on the chain Hochschild complex $C_{\bullet}(A, A)$, $A=C^{\infty}\left(\mathbb{R}^{d}\right)$ (see for details [T], [Sh2], Section 1). Thus, we have two $L_{\infty^{-}}$ modules over $T_{\text {poly }}^{\bullet}\left(\mathbb{R}^{d}\right)$ : these are $C \bullet(A, A)$ and $\Omega^{\bullet}\left(\mathbb{R}^{d}\right)$, the differential forms on $\mathbb{R}^{d}$ with zero differential and usual module structure over $T_{\text {poly }}^{\bullet}$, defined with through the Lie derivatives $L_{\gamma}=i_{\gamma} \circ d \pm d \circ i_{\gamma}$ (see $[\mathrm{T}]$ ).

In [Sh2] we constructed an $L_{\infty}$-morphism of $L_{\infty}$-modules over $T_{\text {poly }}^{\bullet}\left(\mathbb{R}^{d}\right)$, $\hat{\mathcal{U}}: C \bullet(A, A) \rightarrow \Omega^{\bullet}\left(\mathbb{R}^{d}\right)$. Its Taylor components are maps

$$
\hat{\mathcal{U}}_{k}: \Lambda^{k} T_{\text {poly }}^{\bullet}\left(\mathbb{R}^{d}\right) \otimes C \bullet(A, A) \rightarrow \Omega^{\bullet}\left(\mathbb{R}^{d}\right)[-k] .
$$

They are constructed as sums over all admissible graphs, ... etc.

Then for any solution $\pi$ of the Maurer-Cartan equation in $T_{\text {poly }}^{\bullet}\left(\mathbb{R}^{d}\right)$, i.e. of the equation $[\pi, \pi]=0$, one can define the tangent map

$$
T_{\pi} \hat{\mathcal{U}}: T_{\pi} C_{\bullet}(A, A) \rightarrow T_{\pi} \Omega^{\bullet}\left(\mathbb{R}^{d}\right)
$$

where $T_{\pi} C_{\bullet}(A, A)=C_{\bullet}\left(A_{*}, A_{*}\right)$ with $A_{*}$ the Kontsevich deformation quantization, and $T_{\pi} \Omega^{\bullet}\left(\mathbb{R}^{d}\right)=\left\{\Omega^{\bullet}\left(\mathbb{R}^{d}\right), L_{\pi}\right\}$ (see [Sh2], Section 3 for details). The main property of the map $T_{\pi} \hat{\mathcal{U}}$, which follows immediately from the $L_{\infty}$-morphism equations, is that $T_{\pi} \hat{\mathcal{U}}$ is a map of complexes. In degree $0, T_{\pi}^{0} C_{\bullet}(A, A)=A_{*}$ (considered as a vector space), and $T_{\pi}^{0} \Omega^{\bullet}\left(\mathbb{R}^{d}\right)=A$. In degree 0 we obtain a map $T_{\pi} \hat{\mathcal{U}}: A_{*} \stackrel{\sim}{\rightarrow} A$ which is a map of homology, i.e. $T_{\pi} \hat{\mathcal{U}}$ induces a map $A_{*} /\left[A_{*}, A_{*}\right] \stackrel{\sim}{\rightarrow} A /\{A, A\}$ (here $\left[A_{*}, A_{*}\right]$ is the commutant of the deformed algebra, and $\{A, A\}$ is the commutant with respect to the Poisson bracket). See [Sh2], Section 3 for details.

The last property means that $T_{\pi} \hat{\mathcal{U}}$ maps $\left[A_{*}, A_{*}\right]$ to $\{A, A\}$.

Now we are going to prove the following result. 
Theorem. For any Poisson structure $\pi$, any function $\alpha \in A$ such that $[\alpha, \pi]=0$ and any $\beta \in A_{*}$ one has:

$$
\left.T_{\pi} \hat{\mathcal{U}}\left(\left(T_{\pi} \mathcal{U}\right) \alpha\right) * \beta\right)=\alpha \cdot T_{\pi} \hat{\mathcal{U}}(\beta)+c(\alpha, \beta) .
$$

Here $T_{\pi} \mathcal{U}$ is the tangent map with respect to the Kontsevich morphism,

$$
T_{\pi} \mathcal{U}:\left\{T_{\text {poly }}^{\bullet}[1], d=\operatorname{ad} \pi\right\} \rightarrow C^{\bullet}\left(A_{*}, A_{*}\right),
$$

* in the l.h.s. of (6) is the Kontsevich star-product, see [K], Section 8, and $c(\alpha, \beta) \in\{A, A\}$, the Poisson commutant of the algebra $A$.

2.2. First of all, recall the definitions of the tangent maps $T_{\pi} \mathcal{U}, T_{\pi} \hat{\mathcal{U}}$. The case of the Kontsevich formality (i.e. the case of $L_{\infty}$-morphism between dg Lie algebras) is simpler. We have:

$$
\left(T_{\pi} \mathcal{U}\right)(x)=\mathcal{U}_{1}(x)+\mathcal{U}_{2}(x, \pi)+\frac{1}{2} \mathcal{U}_{3}(x, \pi, \pi)+\frac{1}{3 !} \mathcal{U}_{4}(x, \pi, \pi, \pi)+\ldots
$$

It is a map of complexes

$$
T_{\pi} \mathcal{U}:\left\{T_{\text {poly }}^{\bullet}\left(\mathbb{R}^{d}\right)[1], d=\operatorname{ad} \pi\right\} \rightarrow\left\{\mathcal{D}_{\text {poly }}^{\bullet}\left(\mathbb{R}^{d}\right)[1], d=d_{\text {Hoch }}+\operatorname{ad} \tilde{\pi}\right\}
$$

where

$$
\tilde{\pi}=\mathcal{U}_{1}(\pi)+\frac{1}{2} \mathcal{U}_{2}(\pi, \pi)+\frac{1}{3 !} \mathcal{U}_{3}(\pi, \pi, \pi)+\ldots
$$

is the Kontsevich solution of the Maurer-Cartan equation in $\mathcal{D}_{\text {poly }}^{\bullet}\left(\mathbb{R}^{d}\right)$. The last complex can be identified with the complex $C^{\bullet}\left(A_{*}, A_{*}\right)$. More precisely, we should set $\pi:=\hbar \pi$, where $\hbar$ is a formal parameter.

In the case of the Tsygan formality

$$
\left(T_{\pi} \hat{\mathcal{U}}\right)(\omega)=\hat{\mathcal{U}}_{1}(\omega)+\hat{\mathcal{U}}_{2}(\pi, \omega)+\frac{1}{2} \hat{\mathcal{U}}_{3}(\pi, \pi, \omega)+\ldots
$$

It is a map of the complexes

$$
T_{\pi} \hat{\mathcal{U}}: T_{\pi} C_{\bullet}(A, A) \rightarrow T_{\pi} \Omega^{\bullet}\left(\mathbb{R}^{d}\right)
$$

where for an $L_{\infty}$-module $M$ over dg Lie algebra $\mathfrak{g}^{\bullet}$, and a solution $\pi$ of the Maurer-Cartan equation in $\mathfrak{g}^{\bullet}$, the differential in $T_{\pi} M$ is equal to

$$
d \omega=\phi_{0}(\omega)+\phi_{1}(\pi, \omega)+\frac{1}{2} \phi_{2}(\pi, \pi, \omega)+\ldots
$$

where

$$
\phi_{k}: \Lambda^{k} \mathfrak{g}^{\bullet} \otimes M \rightarrow M[1-k]
$$

are the Taylor components of the $L_{\infty}$-module structure. One easily sees that $T_{\pi} C_{\bullet}(A, A) \simeq C_{\bullet}\left(A_{*}, A_{*}\right)$ and $T_{\pi} \Omega^{\bullet}\left(\mathbb{R}^{d}\right) \simeq\left\{\Omega^{\bullet}\left(\mathbb{R}^{d}\right), d=L_{\pi}\right\}$. 


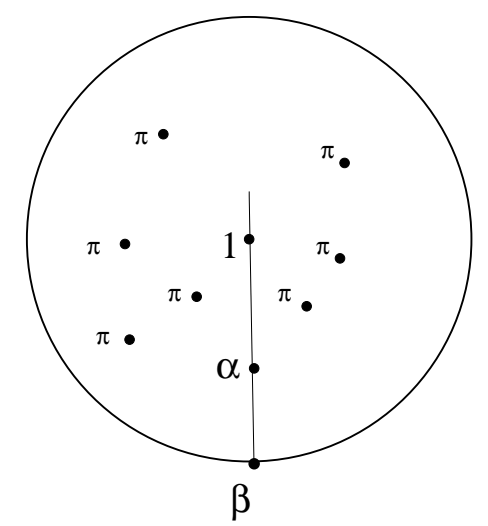

FiguRE 1. A typical configuration we consider

2.2.1. Consider now the disk $D^{2}$ with the center 1 from [Sh2], with only one vertex on the boundary, where is placed $\beta \in A_{*}$, and $n+1$ points inside, in one of them is placed $\alpha \in A=T_{\text {poly }}^{-1}\left(\mathbb{R}^{d}\right)$, and in others are placed copies of $\pi$. We can fix the position of $\beta$ because of the action of the rotation group. Now we consider the configurations where $\alpha \in[\mathbf{1}, \beta]$, see Figure 1 . There is no edge starting at $\mathbf{1}$, because we should obtain a 0 -form.

We consider the sum over all admissible graphs with $2(n+1)+1-2=2 n+1$ edges, i.e. by 1 less that the usual configurations in [Sh2]. But now $\alpha$ moves along the interval $[\mathbf{1}, \beta]$, and the dimension of the configuration space is equal to $2 n+1$. Denote by $D_{1, n+1,1}^{r}$ this configuration space ( $r$ stands for "restricted"), and consider any admissible graph $\Gamma$ with $2 n$ edges. We have:

$$
\int_{\bar{D}_{1, n+1,1}^{r}} d\left(\bigwedge_{e \in E_{\Gamma}} d \varphi_{e}\right)=0
$$

and, by the Stokes formula,

$$
\int_{\partial \bar{D}_{1, n+1,1}^{r}} \bigwedge_{e \in E_{\Gamma}} d \varphi_{e}=0
$$

Now we want to describe the boundary strata in $\partial \bar{D}_{\mathbf{1}, n+1,1}^{r}$ of codimension 1. There are many possibilities. First look for the most interesting:

$\mathbf{S 1 )}$ : the point $\alpha$ and the points $p_{i_{1}}, \ldots, p_{i_{k}}$ of the first type tend to $\mathbf{1}$;

S2): the point $\alpha$ and the points $p_{i_{1}}, \ldots, p_{i_{k}}$ of the first type tend to $\beta \in$ $\partial \bar{D}^{2}=S^{1}$

S3): points $p_{i_{1}}, \ldots, p_{i_{k}}$ of the first type, $p_{i_{s}} \neq \alpha$ for any $s$, tend to $\mathbf{1}$.

There are also other possibilities: 
$\mathbf{S 4 ) : ~ p o i n t s ~} p_{i_{1}}, \ldots, p_{i_{k}}$ of the first type, $p_{i_{s}} \neq \alpha$ for any $s$, tend to $\beta$;

S5): points $p_{i_{1}}, \ldots, p_{i_{k}}, p_{i_{s}} \neq \alpha$ for any $s$, tend to a point $\kappa$ on the boundary, $\kappa \neq \beta$

S6): points $p_{i_{1}}, \ldots, p_{i_{k}}, p_{i_{s}} \neq \alpha$ for any $s$, tend to $\alpha$ and far from $\mathbf{1}$ and from $\beta$;

S7): points $p_{i_{1}}, \ldots, p_{i_{k}}, p_{i_{s}} \neq \alpha$ for any $s$, tend to each other inside the disk.

We have:

$$
\begin{aligned}
& 0=\int_{\partial \bar{D}_{1, n+1,1}}^{r} \bigwedge_{e \in E_{\Gamma}} d \varphi_{e}= \\
& \int_{\left.\partial_{\mathrm{S} 1}\right)} \downarrow+\int_{\partial_{\mathrm{S} 2)}} \downarrow+\int_{\left.\partial_{\mathrm{S} 3}\right)} \downarrow+\int_{\partial_{\mathrm{S} 4)}} \downarrow+\int_{\partial_{\mathrm{S} 5)}} \downarrow+\int_{\partial_{\mathrm{S} 6)}} \downarrow+\int_{\partial_{\mathrm{S} 7)}} \downarrow
\end{aligned}
$$

where $\natural=\bigwedge_{e \in E_{\Gamma}} d \varphi_{e}$.

We claim, that only $\int_{\partial_{\mathrm{S} 1)}} \natural, \int_{\partial_{\mathrm{S} 2}} \downarrow$ and $\int_{\partial_{\mathrm{S} 3)}} \downarrow$ are not equal to 0 , and $\int_{\partial_{\mathrm{S} 1}} \downarrow$ gives exactly first summand of the r.h.s. of $(6), \int_{\left.\partial_{\mathrm{S} 2}\right)} \curvearrowleft$ gives the l.h.s. of (6), and $\int_{\left.\partial_{\mathrm{S} 3}\right)} \downarrow$ gives the second summand in the r.h.s. of $(6), c(\alpha, \beta)$. Therefore, we consider at first these three cases.

2.2.2. The case $S 1)$. By Theorem 6.6 .1 in $[\mathrm{K}]$, the integral over this boundary stratum may not vanish only $k=0$. The situation is like that: only the point $\alpha$ approaches to the point $\mathbf{1}$ along the interval connecting $\mathbf{1}$ and $\beta$. The dimension of this boundary stratum is equal to 0 ; therefore, there should be no edges between 1 and $\alpha$. The picture is like in Figure 2.

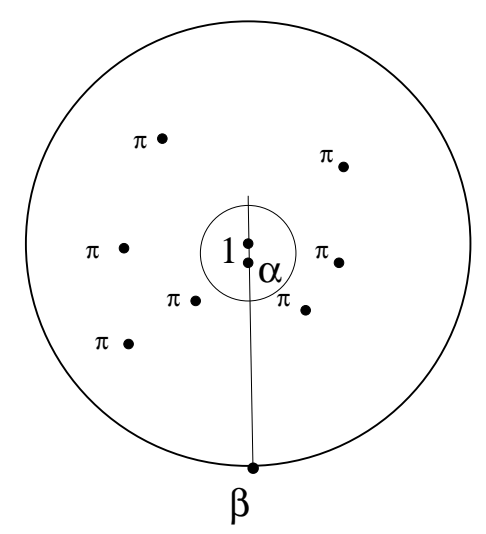

Figure 2. The boundary stratum S1).

This gives exactly $\alpha \cdot T_{\pi} \hat{\mathcal{U}}(\beta)$, i.e. the first summand in the right-hand side of $(6)$. 
2.2.3. The case S2). In this case $\alpha$ approaches to $\beta$. Also, some other points $p_{i_{1}}, \ldots, p_{i_{k}}$ approach to $\beta$. The situation can be described in three steps.

2.2.3.1. At first, we have the Kontsevich-type picture for this boundary stratum. It means that we consider the space $C_{k+1,1}^{r}$ from [K], where $\alpha$ belongs to a vertical line passing through $\beta$ (" $r$ " stands for restricted).

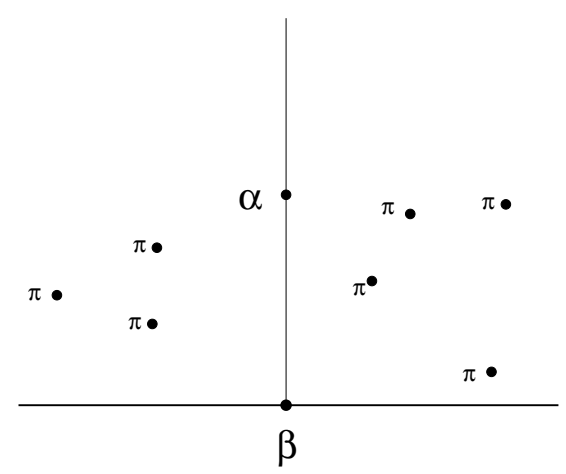

FiguRE 3. The boundary stratum S2). First reduction

The dimension of this stratum is by 1 less than $C_{k+1,1}$, that is it is equal to $2(k+1)+1-2-1=2 k$. Now $\alpha$ is on a finite distance from the boundary. We want to compute the corresponding Kontsevich (poly)differential operator. To do this, we use a second reduction.

2.2.3.2. Now we move $\alpha$ to the boundary.

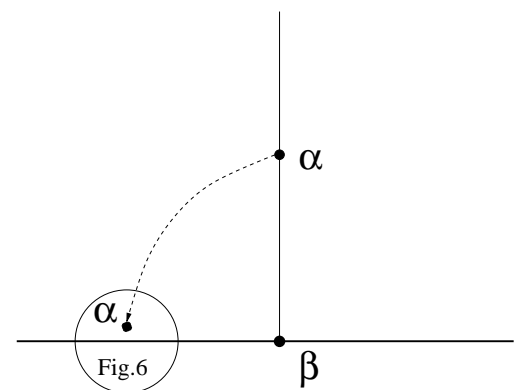

Figure 4. The boundary stratum S2). Second reduction 
In its "final" position, $\alpha$ approaches the boundary, but it still belongs to the interior of the upper half-plane. We obtain a boundary stratum of codimension 1 of $\bar{C}_{k+1,1}$, not $\bar{C}_{k+1,1}$. This boundary stratum has the same dimension that the space $\bar{C}_{k+1}^{r}$, i.e. has a codimension 1 in $\bar{C}_{k+1,1}$. We claim, that polydifferential operators, corresponding to Figure 3 and to Figure 4, coincide. It's again an application of the Stokes formula: certainly, we have some other boundary strata, when several points move close to $\alpha$ in its intermediate position, see Figure 5.

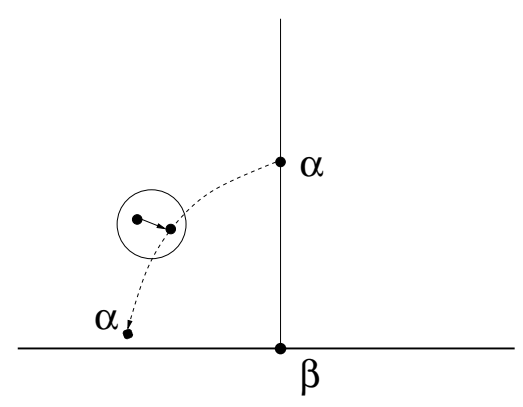

FIgURE 5. An intermediate position of $\alpha$ and a boundary stratum

Some points $p_{j_{1}}, \ldots, p_{j_{l}}$ approaches $\alpha$. By the Theorem 6.6.1 from [K], we have $l=1$. There should be exactly one edge from $p_{j}=p_{j_{1}}$ to $\alpha$. This term corresponds to the bracket $[\pi, \alpha]$, which vanishes because $\alpha$ is supposed to be invariant.

At the picture, showed in Figure 4, we have the polydifferential operator $T(\alpha, \pi) * \beta$, where $*$ is the Kontsevich star-product, and $T(\alpha, \pi)$ is an expression, corresponding to the boundary stratum in Figure 4.

2.2.3.3. The picture for $T(\alpha, \pi)$ is showed in Figure 6 .

It is the usual Kontsevich's picture from $[\mathrm{K}]$. The corresponding function $T(\alpha, \pi)$ is equal to $T_{\pi} \mathcal{U}(\alpha)$. Finally, we see that the expression corresponding to the boundary stratum $\mathrm{S} 2)$, is $T_{\pi} \hat{\mathcal{U}}\left(\left(T_{\pi} \mathcal{U}(\alpha)\right) * \beta\right)$. The $T_{\pi} \hat{\mathcal{U}}$ outside parentheses is corresponded to Figure 3.

2.2.3.4.

Remark. As well we can move $\alpha$ to the right from $\beta$. We will obtain $T_{\pi} \hat{\mathcal{U}}\left(\beta * T_{\pi} \mathcal{U}(\alpha)\right)$. The both expressions coincide because $\alpha$ satisfies $[\pi, \alpha]=0$, and, therefore, $T_{\pi} \mathcal{U}(\alpha)$ is a central element in the deformed algebra. See $[\mathrm{K}]$, Section 8.

2.2.4. The case S3). In this case the boundary stratum is $\bar{D}_{k} \times \bar{D}_{\mathbf{1}, n-k+1,1}^{r}$, where

$$
D_{k}=\left\{p_{1}, \ldots, p_{k} \in \mathbb{C}, p_{i} \neq p_{j} \text { for } i \neq j\right\} /\left\{z \mapsto a z, a \in \mathbb{R}_{>}\right\}
$$




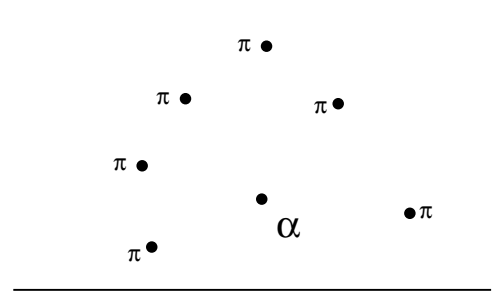

FiguRE 6 . The boundary stratum S2). Third reduction

The space $D_{k}$ has dimension $2 k-1$.It follows from Theorem 6.6.1 in [K] that the integral over $D_{k}$ does not vanish only when $k=1$, and there is no edges from 1 to $p_{1}$. (See [Sh2] for some details). This stratum is corresponded to the second summand, $c(\alpha, \beta)$, in the r.h.s. of (6). At the same time, we obtain an explicit formula for $c(\alpha, \beta)$.

Remark. The stratum S3) here is what was called S2.2) in [Sh2].

2.2.5. Here we consider the remaining cases $\mathrm{S} 4)-\mathrm{S} 7$ ).

2.2.5.1. The case $\mathbf{S} 4)$. In this case the boundary stratum is $\bar{C}_{k, 1} \times \bar{D}_{\mathbf{1}, n-k, 1}^{r}$, it has codimension 1 as expected. The integral factories to the product of $a_{n}$ integral over $\bar{C}_{k, 1}$ and an integral over $\bar{D}_{1, n-k, 1}^{r}$. It is clear that the integral over $\bar{C}_{k, 1}$ vanishes: we attach the bivector field $\pi$ to any point $p_{i_{s}}$, therefore, the number of edges of any graph is $2 k$. But $\operatorname{dim} \bar{C}_{k, 1}=2 k-1$.

Remark. In the case when $\alpha$ also approaches to $\beta$ this argument does not hold, because there are no edges starting at $\alpha$, and $\operatorname{dim} \bar{C}_{k+1,1}^{r}=2 k$.

2.2.5.2. The case S5). The boundary stratum is $\bar{C}_{k, 0} \times \bar{D}_{\mathbf{1}, n-k, 2}^{r}$, it has codimension 1. The integral over $\bar{C}_{k, 0}$ vanishes because any $p_{i}$ is a bivector field, but $\operatorname{dim} \bar{C}_{k, 0}=2 k-2$.

2.2.5.3. The case $\mathbf{S 6 )}$. It is the most principal point that this stratum does not contribute to the integral. By Kontsevich theorem 6.6.1 from [K] we have $k=1$. There is only one edge passing from $p_{i_{1}}$ to $\alpha$, it corresponds to $[\pi, \alpha]=0$ by the assumption.

2.2.5.4. The case S7). Again, $k=2$ by the Theorem 6.6 .1 from [K]. We have $[\pi, \pi]$ which is equal to 0 , because $\pi$ is a Poisson bivector field. 
Theorem 2.1 is proven.

\section{Applications}

For a general Poisson structure $\pi$ on $\mathbb{R}^{d}$, the picture is the following. Denote by $A=C^{\infty}\left(\mathbb{R}^{d}\right)[[h]]$, by $A_{*}$ the Kontsevich deformation quantization of $A$ (with the harmonic angle function). Then, we have two maps:

$$
\begin{aligned}
& T_{\pi} \mathcal{U}: A \rightarrow A_{*} \\
& T_{\pi} \hat{\mathcal{U}}: A_{*} \rightarrow A
\end{aligned}
$$

such that

$$
\left(T_{\pi} \mathcal{U}\right)(\alpha \cdot \beta)=\left(\left(T_{\pi} \mathcal{U}\right) \alpha\right) *\left(T_{\pi} \mathcal{U}(\beta)\right)
$$

for $\alpha, \beta$ such that $[\pi, \alpha]=[\pi, \beta]=0$; in particular, $T_{\pi} \mathcal{U}$ maps the Poisson center to the center of the deformed algebra (for the proof see $[\mathrm{K}]$, Section 8);

$$
T_{\pi} \hat{\mathcal{U}} \operatorname{maps}\left[A_{*}, A_{*}\right] \text { to }\{A, A\}
$$

(for the proof see [Sh2], Section 3);

(iii) compatibility of $T_{\pi} \mathcal{U}$ and $T_{\pi} \hat{\mathcal{U}}$ :

$$
T_{\pi} \hat{\mathcal{U}}\left(T_{\pi} \mathcal{U}(\alpha) * \beta\right)=\alpha \cdot T_{\pi} \hat{\mathcal{U}}(\beta)+c(\alpha, \beta)
$$

here $\alpha \in A,[\pi, \alpha]=0$, and $\beta \in A_{*}$ is arbitrary, and $c(\alpha, \beta) \in\{A, A\}$ (the same that (6), is proven in Theorem 2.1 of the present paper).

Now we are going to consider in more details the case of a linear Poisson structure.

3.1. Let $\pi$ be a linear Poisson structure on $\mathbb{R}^{d} \simeq \mathfrak{g}^{*}, \mathfrak{g}$ is a finite-dimensional Lie algebra, $\pi$ is the Kostant-Kirillov Poisson structure. By definition,

$$
\pi=\sum_{i j k=1}^{\operatorname{dim} \mathfrak{g}} c_{i j}^{k} x_{k} \frac{\partial}{\partial x_{i}} \wedge \frac{\partial}{\partial x_{j}}
$$

where $c_{i j}^{k}$ are the structure constants of the Lie algebra $\mathfrak{g}$ in the basis $\left\{x_{i}\right\}$.

We proved in [Sh1] that $T_{\pi} \mathcal{U}=\mathrm{Id}$ in this case, it is useful (but not necessarily) to use this result here. We have from (16)

$$
T_{\pi} \hat{\mathcal{U}}(\alpha * \beta)=\alpha \cdot T_{\pi} \hat{\mathcal{U}}(\beta)+c(\alpha, \beta)
$$

for any $\beta$ and $\alpha$ such that $[\pi, \alpha]=0$. Here $*$ is the Kontsevich star-product. Now we can suppose that $\mathfrak{g}$ is semisimple and we have the decompositions (4) and (5). Therefore, when we set $\beta=1$ we obtain

$$
T_{\pi} \hat{\mathcal{U}}(\alpha)=\alpha
$$


for any $\alpha$ such that $[\pi, \alpha]=0$. A priori we have from [Sh2]:

$$
T_{\pi} \hat{\mathcal{U}}(f)=\exp \left(\sum_{k \geq 1} w_{2 k} \operatorname{Tr}_{2 k}\right)(f)
$$

for some complex numbers $\left\{w_{2 k}\right\}$. It is enough to know (17) for $\alpha$ such that $[\pi, \alpha]=0$, and for $\mathfrak{g}=\mathfrak{g l}_{n}, n \geq 1$, to conclude that

$$
T_{\pi} \hat{\mathcal{U}}(\alpha)=\alpha \quad \text { for any } \alpha \in A_{(*)} .
$$

The coefficients $\left\{w_{2 k}\right\}$ do not depend on the Lie algebra $\mathfrak{g}$, and we have proved (19) for any $\alpha$ and any Lie algebra $\mathfrak{g}$.

Theorem. For any finite-dimensional Lie algebra $\mathfrak{g}$, any $\alpha \in\left[S^{\bullet}(\mathfrak{g})\right]^{\mathfrak{g}}$ and any $\beta \in S^{\bullet}(\mathfrak{g})$ one has:

$$
\alpha * \beta=\alpha \cdot \beta+c(\alpha, \beta)
$$

where $c(\alpha, \beta) \in\left\{S^{\bullet}(\mathfrak{g}), S^{\bullet}(\mathfrak{g})\right\}$.

Corollary. For any finite-dimensional Lie algebra $\mathfrak{g}$, any $\alpha \in\left[S^{\bullet}(\mathfrak{g})\right]^{\mathfrak{g}}$ and any $\beta \in S^{\bullet}(\mathfrak{g})$ one has:

$$
\varphi_{D}(\alpha \cdot \beta+c(\alpha, \beta))=\varphi_{D}(\alpha) * \varphi_{D}(\beta)
$$

where $\varphi_{D}: S^{\bullet}(\mathfrak{g}) \rightarrow \mathcal{U}(\mathfrak{g})$ is the Duflo map, and $*$ is the product in $\mathcal{U}(\mathfrak{g})$.

Proof. The natural isomorphism of algebras $\Theta: S(\mathfrak{g})_{*} \rightarrow \mathcal{U}(\mathfrak{g})$,

$$
\Theta\left(g_{1} * \cdots * g_{k}\right)=g_{1} \otimes \cdots \otimes g_{k}
$$

is equal to $\varphi_{D}$ (see [Sh1]). We just apply the map $\Theta$ to both sides of (20) and use that $\Theta$ is a map of algebras.

3.2. Remark. It is an interesting question does the Kashiwara-Vergne conjecture $[\mathrm{KV}]$ imply our result in Theorem 3.1. On the other hand, it is interesting does our result (with an explicit form of $c(\alpha, \beta)$ ) opens a way to prove the Kashiwara-Vergne conjecture itself.

\section{Acknowledgements}

Giovanni Felder explained to me the proof of the Kontsevich's theorem on cup-products from $[\mathrm{K}]$, Section 8. Discussions with Boris Feigin were very useful for me. I would like to thank Dominique Manchon for sending me a first version of the paper $[\mathrm{MT}]$. The work was done during my stay at the ETH-Zentrum in remarkable and stimulating atmosphere. I am grateful to IPDE grant 1999-2001 for a particular financial support. 


\section{References}

[AB] D. Arnal, N. Ben Amar, Kontsevich's wheels and invariant polynomial functions on the dual of Lie algebras, to be published in Lett. Math. Phys, 119 (2000).

[ADS] M. Andler, A. Dvorsky, S. Sahi, Kontsevich quantization and invariant distributions on Lie groups, preprint math.QA/9910104.

[CGR] Cahen M., Gutt S., Rawnsley J., On tangential star-products for the coadjoint Poisson structure, Comm. Math. Phys. 180 (1996), 99-108.

[D] M. Duflo, Caractéres des algèbres de Lie résolubles, C. R. Acad. Sci. 269 (1969), 437-438.

[K] M. Kontsevich, Deformation quantization of Poisson manifolds, I, preprint math. q$\operatorname{alg} / 9709040$.

[KV] M. Kashiwara, M. Vergne, The Campbell-Hausdorff fromula and invariant hyperfunctions, Invent. Math., 47 (1978), 249-272.

[MT] D. Manchon, Cohomologie tangente et cup-produit pour la quantification de Kontsevich, Ann. Math. Blaise Pascal, 10 (2003), 75-106.

[T] B. Tsygan, Formality conjecture for chains, math, Amer. Math. Soc. Transl. Ser. 194 (1999), 261-274.

[Sh1] B. Shoikhet, Vanishing of the Kontsevich integrals of the wheels, Lett. Math. Phys. 56 (2001), 141-149.

[Sh2] , A proof of the Tsygan formality conjecture for chains, preprint math. QA/0010321, version2.

FIM, ETH-ZENTRUM, CH-8092 ZüRICH, SWITZERLAND

E-mail address: borya@mccme.ru 